\title{
CARACTERIZACIÓN Y VALORACIÓN ECONÓMICA DE LOS RECHAZOS EN FRONTERA DE PRODUCTOS FRUTÍCOLAS CHILENOS POR INCUMPLIMIENTO DE MEDIDAS NO ARANCELARIAS DE TIPO TÉCNICO
}

\author{
Sofía Boza* y Jazmín Muñoz \\ Institución: Facultad de Ciencias Agronómicas e Instituto de Estudios Internacionales, Universidad de \\ Chile.
}

\begin{abstract}
Resumen
Las medidas no arancelarias (MNA) están muy presentes en la regulación del comercio internacional. Dentro de las MNA técnicas tenemos las medidas sanitarias y fitosanitarias (MSF), las cuales consideran aspectos específicos en calidad e inocuidad alimentaria. El análisis del impacto de las MSF, y las MNA técnicas en general, se ha centrado en sus efectos sobre el comercio mediante modelos gravitacionales. Una forma menos explorada de abordar los efectos de las MSF es el estudio de los rechazos de exportaciones por incumplimiento. El objetivo de la presente contribución es, por tanto, valorizar dichos rechazos, considerando para ello el caso de las exportaciones frutícolas chilenas en el mercado de Estados Unidos. Se aplicó una metodología principalmente cuantitativa, fundamentada en la compilación de bases de datos a partir de información secundaria y primaria (consultas a exportadores), así como en el análisis descriptivo y en la aplicación de una ecuación de valorización original. Los resultados muestran que el valor de los rechazos tiene una participación muy baja en relación con el total de la fruta exportada, lo cual podría deberse al desarrollo del sistema de inocuidad chileno, a un "efecto reputación" fortalecido por los acuerdos comerciales y/o a la no competencia con los productores estadounidenses dada la contra estación.
\end{abstract}

Palabras clave: Medidas Sanitarias y Fitosanitarias, Exportaciones, Fruta, Chile, Estados Unidos.

\section{Introducción}

Las medidas no arancelarias (MNA) están cada vez más presentes en la regulación del comercio internacional (De Melo y Nicita, 2018). Dentro de las MNA, las MSF tienen como objetivo proteger la salud humana, animal y vegetal, regulando aspectos en calidad e inocuidad de productos nacionales e importados. Las MSF están sujetas a regulación multilateral a través del Acuerdo de la Organización Mundial de Comercio sobre la Aplicación de Medidas Sanitarias y Fitosanitarias. Su objetivo es garantizar que los países puedan adoptar y aplicar MSF sin ser una barrera encubierta al comercio (Koul, 2018).

En Chile todavía hay aspectos poco explorados sobre los efectos de las MSF en su comercio exterior, y en especial respecto a los rechazos en frontera. Lo anterior pese a la importancia de la agricultura y la alimentación en su economía: en 2020, el país exportó 17.95 mil millones de dólares estadounidenses en productos agropecuarios y alimentarios, lo que representa más de la mitad de sus exportaciones no cupríferas (Banco Central de Chile, 2021), siendo Estados Unidos uno de sus principales mercados. Allí, la Administración de Alimentos y Medicamentos (FDA) está a cargo de la inspección fronteriza. Esta institución tiene la capacidad para verificar in situ sólo el uno por ciento de los envíos (Artecona y Flores, 2009), por lo que da prioridad a las posibilidades más riesgosas (Elder, 2010). En la decisión de a qué envíos dirigir las inspecciones, la FDA utiliza las notificaciones de entrada documentadas en el Sistema Operativo y Administrativo de Soporte para la Importación (OASIS) (Grundke y Moser, 2019).

En este contexto, el objetivo de esta ponencia es valorizar los rechazos en frontera por incumplimiento con medidas sanitarias y fitosanitarias de las exportaciones frutícolas chilenas a Estados Unidos.

\section{Metodología}

\subsection{Recolección de información sobre rechazos de exportaciones y caracterización}

La información sobre rechazos de exportaciones se recopiló considerando a Chile como exportador y a Estados Unidos como importador. El análisis se centró en fruta fresca (SA-08), debido a que es el producto agrícola más exportado y a que es especialmente sensible a los rechazos fronterizos. La información de rechazos se extrajo de la base de datos de OASIS. El período de estudio fue $2010-2020$. Se utilizaron técnicas de análisis descriptivo para la caracterización de los rechazos de exportaciones.

\subsection{Valorización de rechazos de exportaciones}

La valorización de los rechazos de exportaciones está casi inexplorada. Como consecuencia, se replicó la metodología propuesta de Boza et al. (2018): 


$$
\$ R_{i j t}^{k}=\delta^{f}\left[\frac{C V^{k}}{B V^{k}} * B W^{k}\right] * F O B_{i j t}^{k}
$$

donde $\$ R_{i j t}^{k}$ es el valor económico estimado para un cargamento rechazado con el producto k en el año t, $C V^{k}$ es el volumen del contenedor utilizado para el transporte del producto $\mathrm{k}, B V^{k}$ es el volumen de la caja que se utiliza para transportar el producto $\mathrm{k}, B W^{k}$ es el peso en kilogramos de la caja con la fruta que se utiliza para transportar el producto k, $F O B_{i j t}^{k}$ es el valor FOB promedio por kilogramo exportado del país i al país j para un producto $\mathrm{k}$ en el año t y $\delta^{f}$ es un factor de corrección para el espacio disponible en el contenedor para pallets, carga, descarga y ventilación, en tanto por uno. La información sobre logística de transporte se consultó con empresas y asociaciones de exportadores. La información respecto al valor FOB de exportaciones se extrajo de estadísticas de comercio exterior de ODEPA (2021). Se asumió tras consultas expertas realizadas que un envío rechazado equivale a un contenedor.

\subsection{Comparación del valor de rechazos con las exportaciones totales}

El valor monetario estimado de rechazos se dividió por el valor FOB de exportaciones chilenas de productos SA-08 hacia Estados Unidos. El resultado se utilizó para mostrar el valor relativo de los rechazos respecto al total de las exportaciones de fruta fresca.

\section{Resultados}

Durante el periodo estudiado, el número de envíos chilenos rechazados fue de específicamente 400 contenedores. Tal como muestra el Cuadro 1, la principal razón de rechazo fue la existencia de residuos de pesticidas, lo que sugiere que en dichos casos no se ha respetado el periodo de carencia de las aplicaciones en campo. Además, la mayor concentración de rechazos ocurre en 2019, cuando se le prohibió la entrada a 184 contenedores de limones, que correspondieron principalmente a un solo proveedor (153 envíos). Le siguen 2014, con 47 rechazos, 2011 con 32 y 2020 con 26. Otras razones de rechazos destacadas son la presencia de suciedad y/o descomposición y la contaminación por listeria.

Cuadro 1. Número de envíos chilenos rechazados en frontera estadounidense por tipo de incumplimiento

\begin{tabular}{|c|c|c|c|c|c|c|c|c|c|c|c|}
\hline & 2010 & 2011 & 2012 & 2013 & 2014 & 2015 & 2016 & 2017 & 2018 & 2019 & 2020 \\
\hline Pesticidas & 2 & 12 & 10 & 18 & 37 & 5 & 5 & 1 & 4 & 195 & 20 \\
\hline Suciedad/Descomposición & & 18 & 1 & 2 & 8 & & 1 & & 6 & 2 & \\
\hline Insalubridad & & 1 & & & & & & & & $6^{+}$ & \\
\hline Toxicidad & & & & & & & & & & & 5 \\
\hline Listeria & 1 & & & & & & & & & $33^{x}$ & 1 \\
\hline Aditivos inseguros (color) & & 1 & 1 & & & & & & & & \\
\hline Etiquetado & & & & 2 & 2 & & & & & & \\
\hline Total & 3 & 32 & 12 & 22 & 47 & 5 & 6 & 1 & 10 & 236 & 26 \\
\hline
\end{tabular}

+Insalubridad y listeria; ${ }^{\times}$Listeria y pesticidas

Fuente: Elaboración propia con datos de OASIS

En cuanto a los productos agrícolas involucrados, estos abarcan una gran variedad. Limones y nectarines son los que presentan mayor número de rechazos, cuya causa es la existencia de pesticidas; y pasas, en las que la razón de rechazo se involucra a la presencia de suciedad (Cuadro 2). No obstante, se destaca el bajo número de rechazos de los productos agrícolas chilenos más importados por Estados Unidos en valor: uva de mesa y arándanos, con un acumulado de dos y seis contenedores respectivamente. 
Cuadro 2. Número de envíos chilenos rechazados en frontera estadounidense por producto

\begin{tabular}{|c|c|c|c|c|c|c|c|c|c|c|c|}
\hline Producto & 2010 & 2011 & 2012 & 2013 & 2014 & 2015 & 2016 & 2017 & 2018 & 2019 & 2020 \\
\hline Almendras con cáscara & & 1 & 1 & & 3 & & & & & & \\
\hline Arándanos & & & 3 & 2 & 1 & & & & & & \\
\hline Berries deshidratados o pasta, sin clasificar & 1 & 2 & 3 & 1 & 2 & & & & & & \\
\hline Berries, sin clasificar & & & & 1 & 2 & & & & & & \\
\hline Ciruelas & & 1 & & & & & & & & 6 & 4 \\
\hline Clementinas & & & & & & & & & 1 & & 4 \\
\hline Duraznos (melocotones) & & & & 3 & & 1 & 2 & 1 & 2 & 6 & \\
\hline Frambuesas & 1 & & & 8 & 10 & & & & & & \\
\hline Frutillas (fresas) & & & & & & & 1 & & & & 7 \\
\hline Frutillas (fresas) deshidratadas & & & 2 & & 1 & & & & & & \\
\hline Higos & & & 1 & & & & & & & & \\
\hline Limones & & & & & & & & & & 184 & 6 \\
\hline Moras & & 4 & & & 1 & 1 & & & & 1 & 1 \\
\hline Naranjas & & & 1 & & & & & & & & \\
\hline Nectarines & & 1 & & 2 & 22 & 3 & 1 & & & 36 & 4 \\
\hline Paltas (avocados) & 1 & & & & & & & & & & \\
\hline Pasas & & 18 & 1 & 5 & 5 & & 1 & & 6 & 3 & \\
\hline Peras & & 5 & & & & & & & & & \\
\hline Uvas & & & & & & & 1 & & 1 & & \\
\hline
\end{tabular}

Fuente: Elaboración propia con datos de OASIS

Con respecto a la valorización económica de los rechazos, el cálculo se realizó utilizando la fórmula descrita en 2.2. El coeficiente $\delta^{f}$ utilizado fue 0,8 . Se estandarizó el tamaño de contenedor para fruta fresca a 40 pulgadas (refrigerado) y para productos de agroindustria a 20 pulgadas, con un volumen aproximado de 60,20 y 26,95 metros cuadrados respectivamente (TIS-GDV, 2020). Se excluyeron los productos de corta postcosecha: moras, frambuesas y fresas, ya que su transporte en fresco es aéreo, por lo que la propuesta no estima correctamente el valor monetario de sus rechazos. También se excluyó un envío de manzanas deshidratadas y uno de pasta de membrillo, ya que no es posible realizar su valorización con la información existente. Sin embargo, se espera que su exclusión no sea significativa, dada la baja importancia relativa de dichos productos en el valor total exportado.

El valor estimado de los rechazos es de 9.451.100,94 de dólares estadounidenses, lo que representa un 0,046 por ciento de las exportaciones totales de fruta chilena a Estados Unidos durante 2010-2020. Dichas exportaciones totales contabilizaron cerca de 10.500 toneladas de fruta (SA-08) por un valor FOB aproximado de 20.550 millones de dólares. Esto señala a Estados Unidos como el socio comercial más importante para Chile en fruta durante el periodo estudiado.

También se observa que la valorización de los rechazos por año es concordante con el número de envíos. Como se muestra en el Cuadro 3, el mayor valor se tiene en 2019, cuando existió un alto número de contenedores de limones rechazados, como se discutió previamente.

Cuadro 3. Valorización de envíos chilenos rechazados en frontera estadounidense por año

\begin{tabular}{|c|c|c|c|c|c|c|c|c|c|c|c|}
\hline & 2010 & 2011 & 2012 & 2013 & 2014 & 2015 & 2016 & 2017 & 2018 & 2019 & 2020 \\
\hline Valor (US\$) & 126.19 & 781.946 & 506.829 & 557.012 & 1.516 .220 & .154 & 141.533 & 25.385 & 16.658 & 4.933.394 & 456.779 \\
\hline
\end{tabular}

Fuente: Elaboración propia

Los datos disponibles sobre rechazos de envíos no informan sobre el destino final de los contenedores, por lo tanto, no es posible determinar si estos corresponden a pérdidas totales o si son redestinados. No obstante, el valor de los rechazos es relativamente bajo, lo que coincide con los resultados de Boza et al. (2018).

\section{Conclusiones}

Las investigaciones sobre rechazos en frontera por incumplimiento con MSF son escasas y se han centrado principalmente en describir el fenómeno, pero no en su valorización. La evidencia existente señala que los productos perecederos, especialmente frutas y verduras, son los más susceptibles de ser rechazados (Bovay, 2016; Artecona y Flores, 2009; Brooks et al., 2009); así como los importados de países en desarrollo (Buzby y Roberts, 2011). La reputación del exportador relacionada con su historial de (in)cumplimiento es una variable significativa que motiva (o inhibe) los rechazos futuros (Taghouti et al., 2016; Jouanjean et al., 2015; Jouanjean, 2012). Esto podría indicar que cada rechazo aumenta el riesgo sanitario percibido, lo que según Jaud et al. (2013) conduce a la concentración a favor de ciertos exportadores. En este sentido, Chile 
se posiciona como un país con bajo número de rechazos de productos frutícolas en la frontera estadounidense, los que valorizados entre 2010 y 2020 representan menos del 0,05 por ciento de las exportaciones totales de fruta chilena a Estados Unidos. Lo anterior sugiere que tanto la regulación en MSF de Chile como su sistema de control (sobre todo en exportación) son destacables. Además, la gran cantidad de acuerdos comerciales firmados por Chile podría explicar en parte la buena reputación que goza, ya que a través de ellos se establecen nexos de colaboración e intercambio de información que incluyen las MSF (de hecho, con Estados Unidos existen mesas de diálogo conjuntas y presencia del USDA en los puntos de control de la fruta). Así mismo, esta apertura comercial podría incidir sobre la regulación de MSF chilena, buscando una homogeneización con la de importadores clave como Estados Unidos. Por otro lado, el no competir directamente con la producción estadounidense, dado que los productos chilenos aprovechan las "ventanas" comerciales que les da la contraestación, también puede ser un factor relevante.

\section{Bibliografía}

Artecona, R. y Flores, F.A. (2009). Observatorio del control aduanero a las importaciones de Estados Unidos: estándares técnicos. CEPAL, Washington, D.C.

Banco Central de Chile. (2021). Indicadores de Comercio Exterior cuarto trimestre 2020. Disponible en https://www.bcentral.cl/en/content/-/details/indicadores-de-comercio-exterior-cuarto-trimestre-2020

Bovay, J. (2016). FDA Refusals of Imported Food Products by Country and Category, 2005-2013. Economic Information Bulletin No. 151. United States Department of Agriculture, Economic Research Service, Washington, D.C.

Boza, S., Rivers, R., Rozas, J. y Muñoz, J. (2018). Implications of Non-Compliance with Technical NonTariff Measures: The Case of Chilean Food Related Export Refusals at the United States Border. En: De Melo, J. y Nicita, A. (Eds.): Non-Tariff Measures: Economic Assessment and Policy Options for Development. United Nations Publications, Geneva: 225-253.

Brooks, N., Buzby, J.C. y Regmi, A. (2009). Globalization and evolving preferences drive U.S. food-import growth. Journal of Food Distribution Research, 40:39-46.

Buzby, J.C. y Roberts, D. (2011). Food trade and food safety violations: What can we learn from import refusal data? American Journal of Agricultural Economics, 93:560-565.

De Melo, J. y Nicita, A. (2018). Non-Tariff Measures: Economic Assessment and Policy Options for Development. United Nations Publications, Geneva.

Elder, D. (2010). Ensuring the safety of imported products. Consumer health information. Department of Agriculture, United States.

Engler, A., Nahuelhual, L., Cofré, G. y Barrena, J. (2012). How far from harmonization are sanitary, phytosanitary and quality-related standards? An exporter's perception approach. Food Policy, 37:162-170. Grundke, R. y Moser, C. (2019). Hidden protectionism? Evidence from non-tariff barriers to trade in the United States. Journal of International Economics, 117: 143-157.

Jaud, M., Cadot, O. y Suwa-Eisenmann, A. (2013). Do food scares explain supplier concentration? An analysis of EU agri-food imports. European Review of Agricultural Economics, 40(5): 873-890.

Jouanjean, M.A. (2012). Standards, reputation, and trade: evidence from US horticultural import refusals. World Trade Review, 11:438-461.

Jouanjean, M. A., Maur, J.C. y Shepherd, B. (2015). Reputation matters: Spillover effects for developing countries in the enforcement of US food safety measures. Food Policy, 55: 81-91.

Koul, A.K. (2018). Guide to the WTO and GATT: Economics, Law and Politics. Berlin, Germany: Springer. Oficina de Estudios y Políticas Agrarias (ODEPA). (2021). Bases de datos comercio exterior. Sistema Matriz detallada de comercio exterior. Disponible en https://www.odepa.gob.cl/estadisticas-delsector/bases-de-datos-comercio-exterior

Taghouti, I., Martínez-Gómez, V. y Marti, L. (2016). Sanitary and Phytosanitary measures in agri-food imports from the European Union: Reputation effects over time. Economía Agraria y Recursos Naturales, 16(2): 69-88.

Transport Information Service - The German Insurance Association (TIS-GDV). (2020). Refrigerated and insulated containers. Disponible en https://www.tis-gdv.de/tis_e/containe/arten/kuehlcon/kuehlcon.htm/ 